\title{
DISPONIBILIDADE DE COBRE E ZINCO EM SOLOS DO SUL DO BRASIL ${ }^{(1)}$
}

\author{
Leandro Bortolon $^{(2)} \&$ Clesio Gianello $^{(3)}$
}

\begin{abstract}
RESUMO
O estudo da eficiência de soluções extratoras multielementares para avaliação da disponibilidade de nutrientes do solo às plantas tem aumentado. $O$ uso dessas soluções aumenta a eficiência dos laboratórios de análise de solo devido à redução de procedimentos analíticos. Além disso, a determinação de nutrientes por espectrometria de emissão ótica por plasma induzido (ICP-OES) tem aumentado nos últimos anos, visando principalmente à redução dos procedimentos analíticos e dos limites de deteç̧ão. Com os objetivos de selecionar métodos de extração e verificar a eficiência para avaliar a disponibilidade de $\mathrm{Cu}$ e $\mathrm{Zn}$ para as plantas, foram realizados estudos em casa de vegetação e em laboratório. Em casa de vegetação, amostras de solos representativos do Estado do Rio Grande do Sul foram cultivadas, em vasos de $11 \mathrm{dm}^{3}$ (a céu aberto), com plantas de milho (Zea mays), por $45 \mathrm{~d}$ e, em sequência, com soja (Glycine max), por mais $45 \mathrm{~d}$. Os métodos estudados para extração de $\mathrm{Zn}$ e Cu foram: $\mathrm{HCl} \mathrm{0,1}$ mol L $^{-1}(\mathrm{HCl})$, Mehlich-1 $\left(\mathrm{M}_{1}\right)$ e Mehlich-3 $\left(\mathbf{M}_{3}\right)$. Os coeficientes de determinação obtidos entre as quantidade de $\mathrm{Cu}$ absorvida pelas plantas e o extraído pelos diferentes métodos foram: $\mathrm{HCl}=$ $0,56^{* *}, M_{1}=0,35^{*} ; M_{3}=0,46^{* *}$ para as plantas de milho; $\mathrm{e} \mathrm{HCl}=0,66^{* *}, \mathrm{M}_{1}=0,43^{*}, \mathrm{M}_{3}$ $=0,93^{* *}$ para as de soja. Para o $\mathrm{Zn}$ absorvido pelas plantas e o extraído pelos diferentes métodos, foram: $\mathrm{HCl}=0,18^{*}, M_{1}=0,32^{* *}, M_{3}=0,10^{*}$ e $\mathrm{HCl}=0,37^{*}, M_{1}=$ $0,36^{* *}, M_{3}=0,31^{*}$, respectivamente para as plantas de milho e de soja. Os métodos tiveram baixa capacidade preditiva na avaliação da disponibilidade de $\mathrm{Cu}$ e de $\mathrm{Zn}$ para as plantas.
\end{abstract}

Termos de indexação: análise de solo, correlação, micronutrientes.

\footnotetext{
(1) Parte da Dissertação de mestrado do primeiro autor apresentada ao Programa de Pós-Graduação em Ciência do Solo, Universidade Federal do Rio Grande do Sul - UFRGS. Pesquisa realizada com apoio financeiro do Projeto Laboratório de Análises de Solos do Departamento de Solos e do CNPq. Recebido para publicação em julho de 2008 e aprovado em fevereiro de 2009.

(2) Engenheiro-Agrônomo, Aluno do Programa de Pós-Graduação em Ciência do Solo da Universidade Federal do Rio Grande do Sul - UFRGS. Av. Bento Gonçalves 7712, CEP 91540-000 Porto Alegre (RS). E-mail: leandro.bortolon@ufrgs.br

${ }^{(3)}$ Professor Associado do Departamento de Solos, UFRGS. E-mail: cgianello@hotmail.com
} 


\title{
SUMMARY: AVAILABILITY OF COPPER AND ZINC IN SOILS FROM SOUTHERN BRAZIL
}

\begin{abstract}
The study of the efficiency of multielement extractant solutions to assess the availability of soil nutrients for plants has increased recently. The use of these solutions increases the efficiency of soil analysis laboratories by simplifying analytical procedures. Furthermore, the determination of nutrients by Inductively Coupled Plasma Optical Emission spectrometry (ICP-OES) has increased in recent years, owing mainly to simpler test procedures and less limitations in the detection of elements. Aiming at evaluating methods of extraction and verifying their efficiency to assess $C u$ and $Z n$ availability to plants, greenhouse and laboratory studies were conducted. In the greenhouse study, representative soil samples of the state of Rio Grande do Sul were accommodated in $11 \mathrm{dm}^{3}$ pots and cultivated with corn (Zea mays) plants for $45 \mathrm{~d}$, and soon after with soybean (Glycine max) for another $45 \mathrm{~d}$. The extraction methods for Zn and Cu were: $\mathrm{HCl} 0.1$ mol L-1 (HCl), Mehlich-1 (M1) and Mehlich-3 (M3). The determination coefficients between the $C u$ amounts absorbed by plants and amounts extracted by the different methods were: $\mathrm{HCl}=0.56^{* *}, M_{1}=0.35^{*}, M_{3}=0.46^{* *}$ for corn plants and $\mathrm{HCl}=0.66^{* *}, M_{1}=0.43^{*}, M_{3}$ $=0.93^{* *}$ for soybean. For the zinc amount taken up by plants and that extracted by various methods the determination coefficients were: $\mathrm{HCl}=0.18^{*}, M_{1}=0.32^{* *}, M_{3}=0.10^{*}$ and $\mathrm{HCl}=$ $0.37^{*}, M_{1}=0.36^{* *}, M_{3}=0.31^{*}$, for corn and soybean plants, respectively. The predictive capacity of the methods in assessing the availability of $\mathrm{Cu}$ and $\mathrm{Zn}$ for plants was low.
\end{abstract}

Index terms: soil text, correlation, micronutrients.

\section{INTRODUÇÃO}

A produtividade agrícola pode ser limitada em algumas regiões do Brasil pela deficiência de micronutrientes decorrente da baixa fertilidade natural desses solos, da maior remoção nas colheitas e do uso crescente de corretivo da acidez aplicado em superfície, principalmente no sistema plantio direto. Além disso, a maior pureza nos fertilizantes NPK, que deixam de fornecer micronutrientes como impurezas, tem contribuído para a ocorrência de deficiências de micronutrientes no solo.

O conhecimento da disponibilidade dos micronutrientes no solo é fundamental para uma recomendação de adubação adequada, evitando assim problemas de deficiência ou de toxidez. Na seleção de um método de análise de solo, é fundamental que haja relação positiva entre a concentração do nutriente extraído pelo método e o acúmulo do nutriente nas plantas.

No Brasil, a disponibilidade de $\mathrm{Cu}$ e de $\mathrm{Zn}$ é avaliada, principalmente, por soluções ácidas diluídas, como a solução de $\mathrm{HCl} 0,1 \mathrm{~mol} \mathrm{~L}^{-1}$ (Wear \& Somer, 1948) e a solução de Mehlich-1 $\left(\mathrm{M}_{1}\right)\left(\mathrm{HCl} 0,05 \mathrm{~mol} \mathrm{~L}^{-1} \mathrm{e}\right.$ $\mathrm{H}_{2} \mathrm{SO}_{4} 0,0125 \mathrm{~mol} \mathrm{~L}^{-1}$ ) (Mehlich, 1953). No entanto, pode ser avaliada por soluções ácidas diluídas com adição de agente complexante, como a solução de Mehlich-3 $\left(\mathrm{M}_{3}\right)\left(\mathrm{CH}_{3} \mathrm{COOH} 0,02 \mathrm{~mol} \mathrm{~L}^{-1}, \mathrm{NH}_{4} \mathrm{NO}_{3}\right.$ $0,25 \mathrm{~mol} \mathrm{~L}^{-1}, \mathrm{NH}_{4} \mathrm{~F} 0,015 \mathrm{~mol} \mathrm{~L}^{-1}, \mathrm{HNO}_{3} 0,013 \mathrm{~mol} \mathrm{~L}^{-1}$ e EDTA 0,001 $\mathrm{mol} \mathrm{L}^{-1}$ ) (Mehlich, 1984), que tem capacidade de extrair simultaneamente vários elementos em um único procedimento analítico.

$\mathrm{O}$ método de avaliação da disponibilidade de $\mathrm{Cu}$ e de Zn para as plantas utilizado pela Rede Oficial de
Laboratórios de Análise de Solo dos Estados do Rio Grande do Sul e Santa Catarina é a solução extratora de $\mathrm{HCl} \mathrm{0,1} \mathrm{mol} \mathrm{L}{ }^{-1}$ (CFSR/SC, 2004). Os coeficientes de determinação entre os valores de $\mathrm{Cu}$ e de $\mathrm{Zn}$ extraídos por essa solução e o acúmulo destes pelas plantas são altos (Lantmann \& Meurer, 1982; Bataglia \& Raij, 1989, 1994; Buzetti, 1992; Oliveira et al., 1999; Pereira et al., 2001). Além disso, os teores obtidos são grandes, o que facilita a utilização desses extratores em laboratórios de análise de solo. Contudo, nesses laboratórios há necessidade de vários extratores para determinação dos elementos no solo, reduzindo a eficiência analítica. A utilização de extrator multielementar pode aumentar essa eficiência.

Vários trabalhos reportam a eficiência da solução de Mehlich-1 $\left(\mathrm{M}_{1}\right)$ e de Mehlich-3 ( $\left.\mathrm{M}_{3}\right)$ na avaliação da disponibilidade de $\mathrm{Cu}$ e de $\mathrm{Zn}$ para as plantas, com teores extraídos semelhantes ao $\mathrm{HCl}$ 0,1 $\mathrm{mol} \mathrm{L}^{-1}(\mathrm{HCl})$ e com alto grau de relação com a absorção pelas plantas (Lantmann \& Meurer, 1982; Bataglia \& Raij, 1989, 1994; Barbosa Filho et al., 1990; Buzetti, 1992; Davis et al., 1995; Abreu \& Raij, 1996; Oliveira et al., 1999; Cancela et al., 2001; Borkert et al., 2004).

O objetivo deste estudo foi comparar os métodos HCl 0,1 mol L-1, Mehlich-1 e Mehlich-3 na avaliação da disponibilidade de $\mathrm{Cu}$ e de $\mathrm{Zn}$ para as culturas do milho e da soja em solos do Estado do Rio Grande do Sul.

\section{MATERIAL E MÉTODOS}

O presente trabalho foi realizado para avaliar a disponibilidade de $\mathrm{Cu}$ e de $\mathrm{Zn}$ para as plantas em 
diferentes solos do Estado do Rio Grande do Sul, a céu aberto, nas instalações do Departamento de Solos da Faculdade de Agronomia da UFRGS, comparando a eficiência de soluções extratoras na avaliação da disponibilidade de $\mathrm{Cu}$ e de $\mathrm{Zn}$ do solo para as plantas.

Foram utilizadas amostras de solos - com ampla variação de suas características físicas, químicas e mineralógicas - das classes mais representativas do Estado do RS (Quadro 1), coletadas por Kroth (1998) (Quadro 2). Foram utilizadas amostras de 12 solos no primeiro cultivo e de nove no segundo, quando foram excluídas as amostras dos solos das unidades Vacaria, Bom Jesus e Aceguá, por apresentarem limitações ao crescimento e desenvolvimento das plantas de soja, devido à baixa disponibilidade de nutrientes após o cultivo do milho.

Os solos foram passados em peneira de malha com orifícios de $4 \mathrm{~mm}$ de diâmetro. Foram adicionados por $\mathrm{dm}^{-3}$ de solo: $0,1 \mathrm{mg}$ de $\mathrm{Mo}\left(\left(\mathrm{NH}_{4}\right)_{6} \mathrm{Mo}_{7} \mathrm{O}_{24} \cdot 4 \mathrm{H}_{2} \mathrm{O}\right)$, $0,5 \mathrm{mg}$ de $\mathrm{B}\left(\mathrm{H}_{3} \mathrm{BO}_{3}\right)$ e $5,0 \mathrm{mg}$ de $\mathrm{S}\left((\mathrm{NH} 4)_{2} \mathrm{SO}_{4}\right)$, em solução. Não houve necessidade de correção da acidez dos solos em estudo, pois os teores de $\mathrm{Al}$ trocável estavam abaixo dos valores-limite para alterar o crescimento radicular. Além disso, não houve necessidade de adição do $\mathrm{P} \mathrm{e} \mathrm{K}$, pois os teores desses nutrientes nos solos estavam enquadrados nas classes alto ou muito alto (CFSR/SC, 2004).

Após a adição desses nutrientes, as amostras de solos foram novamente homogeneizadas. Foram, então, preparadas as unidades experimentais, constituídas de colunas de solo em tubos de PVC de $30 \mathrm{~cm}$ de altura, com capacidade para $11 \mathrm{dm}^{3}$. Na parte inferior dos vasos foi colocado um suporte perfurado e uma tela de náilon com ocupação equivalente a $1 \mathrm{dm}^{3}$, para facilitar a drenagem. Um volume de $9 \mathrm{dm}^{3}$, de cada amostra de solo, foi transferido para o tubo de PVC. Os tratamentos (tipo de solo) foram dispostos em blocos casualizados, com três repetições. Durante 70 d, os tubos de PVC foram mantidos ao tempo, cobertos com uma tela de náilon para evitar o impacto direto das gotas da chuva na superfície do solo. Na ausência de chuva, os solos foram irrigados com água deionizada em intervalos de 4 a 5 d, para manter o solo com umidade entre 75 e $85 \%$ da capacidade de campo.

Antes da semeadura das culturas do milho e da soja, foram coletadas amostras de solo da camada superficial (aproximadamente de 0 a $20 \mathrm{~cm}$ de profundidade), de aproximadamente $0,25 \mathrm{dm}^{3}$, utilizando um trado calador. Após a coleta, a amostra de solo, contida em cada unidade experimental (na camada de $0-20 \mathrm{~cm}$ ), foi revolvida, com a finalidade de homogeneização, e compactada levemente (esse procedimento foi feito antes de cada cultivo). As amostras de solo foram secas em estufa com circulação forçada de ar, durante $72 \mathrm{~h}$, à temperatura de $45^{\circ} \mathrm{C}$. Posteriormente, o solo foi moído em amofariz de porcelana e tamisado em peneira com orifícios de $2 \mathrm{~mm}$ de diâmetro.

Nas colunas de solo, no primeiro cultivo, foi semeado milho (Zea mays), utilizando-se seis sementes por vaso. Após a emergência das plântulas, foi feito o desbaste, mantendo-se três plantas por vaso. A adubação nitrogenada foi feita com solução de ureia, aplicada na superfície do solo, na dose de $100 \mathrm{mg} \mathrm{dm}^{-3}$ de N, parcelada em três aplicações, sendo 1/3 na semeadura, $1 / 3$ aos $15 \mathrm{~d}$ e $1 / 3$ aos $30 \mathrm{~d}$ da semeadura. Após $45 \mathrm{~d}$ de cultivo, as plantas foram cortadas pelo caule, a $1 \mathrm{~cm}$ da superfície do solo, e secas em estufa à temperatura de $65^{\circ} \mathrm{C}$, até massa constante.

Quadro 1. Classificação, material de origem e locais de coleta das amostras de solos utilizadas no experimento

\begin{tabular}{lll}
\hline \multicolumn{1}{c}{ Classificação brasileira ${ }^{(1)}$} & Unidade de mapeamento & Material de origem \\
\hline & & Município de coleta \\
Vertissolo Ebânico órtico chernossólico & Aceguá & Siltito \\
Planossolo Háplico eutrófico vértico & Bagé & Siltito \\
Cambissolo Húmico alumínico típico & Bom Jesus & Eruptiva básica \\
Chernossolo Argilúvio férrico típico & Ciríaco & Eruptiva básica \\
Latossolo Vermelho distrófico típico & Cruz Alta & Arenito \\
Latossolo Vermelho aluminoférrico típico & Erechim & Eruptiva básica \\
Nitossolo Vermelho distrófico latossólico & Estação & Eruptiva básica \\
Argissolo Vermelho-Amarelo alumínico típico & Júlio de Castilhos & Eruptiva básica \\
Latossolo Vermelho distrófico típico & Passo Fundo & Erup. básica e arenito \\
Argissolo Vermelho distrófico típico & São Jerônimo de Castilhos & Erechim \\
Chernossolo Ebânico carbonático vértico & Uruguaiana & Granito \\
Latossolo Bruno alumínico câmbico & Vacaria & Sedimento de basalto \\
\hline
\end{tabular}

Fonte: Kroth (1998); Embrapa (1999). 


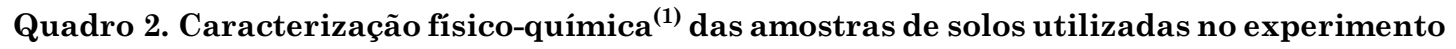

\begin{tabular}{|c|c|c|c|c|c|c|c|c|c|c|c|}
\hline $\begin{array}{c}\text { Unidade de } \\
\text { Mapeamento }^{(2)}\end{array}$ & Argila & pH $\mathrm{H}_{2} \mathrm{O}$ & Índice SMP & MO & $\mathbf{P}$ & $\mathbf{K}$ & $\mathrm{Zn}$ & $\mathbf{C u}$ & $\mathbf{C a}^{2+}$ & $\mathrm{Mg}^{2+}$ & $\mathrm{Al}^{3+}$ \\
\hline & $\%$ & & & $\mathrm{~g} \mathrm{~kg} \cdot 1$ & \multicolumn{4}{|c|}{$-\mathrm{mg} \mathrm{dm}^{-3}$} & \multicolumn{3}{|c|}{$-\mathrm{cmol}_{\mathrm{c}} \mathrm{dm}^{-3}-$} \\
\hline Cruz Alta & 25 & 5,7 & 6,4 & 17 & 36,6 & 81 & 2,3 & 2,3 & 3,4 & 0,9 & 0,0 \\
\hline Vacaria & 50 & 5,9 & 6,1 & 48 & 11,4 & 545 & 6,7 & 10,7 & 8,7 & 2,7 & 0,0 \\
\hline Uruguaiana & 25 & 5,5 & 6,2 & 31 & 14,4 & 107 & 4,5 & 5,1 & 11,0 & 3,8 & 0,0 \\
\hline Bom Jesus & 13 & 6,5 & 6,1 & 107 & 12,4 & 228 & 0,7 & 0,2 & 16,8 & 3,6 & 0,0 \\
\hline Bagé & 20 & 5,5 & 6,1 & 24 & 44,4 & 136 & 2,6 & 2,1 & 6,1 & 2,8 & 0,0 \\
\hline Aceguá & 32 & 5,7 & 6,2 & 46 & 28,2 & 355 & 5,6 & 2,4 & 11,8 & 4,4 & 0,0 \\
\hline São Jerônimo & 24 & 5,4 & 6,4 & 20 & 27,3 & 103 & 3,6 & 3,1 & 2,6 & 0,8 & 0,4 \\
\hline Passo Fundo & 28 & 5,6 & 6,0 & 26 & 28,1 & 113 & 4,1 & 4,1 & 4,9 & 1,2 & 0,0 \\
\hline Ciríaco & 40 & 5,8 & 6,0 & 37 & 12,8 & 316 & 7,8 & 5,2 & 14,3 & 4,9 & 0,0 \\
\hline Júlio de Castilhos & 41 & 5,5 & 6,0 & 28 & 18,3 & 248 & 6,0 & 4,0 & 5,2 & 1,5 & 0,0 \\
\hline Erechim & 53 & 5,7 & 6,0 & 38 & 15,3 & 174 & 6,0 & 7,0 & 7,7 & 1,8 & 0,0 \\
\hline Estação & 43 & 5,7 & 6,1 & 29 & 18,8 & 289 & 6,0 & 5,2 & 5,9 & 1,3 & 0,0 \\
\hline
\end{tabular}

${ }^{(1)}$ Tedesco et al. (1995). ${ }^{(2)}$ Embrapa (1999).

No segundo cultivo foi semeada soja (Glycine max), utilizando-se, por vaso, seis sementes inoculadas com rizóbio. Após a emergência das plantas, foi feito o desbaste, mantendo-se três plantas por vaso. Aos $45 \mathrm{~d}$ de cultivo, as plantas foram cortadas a $1 \mathrm{~cm}$ da superfície do solo e secas em estufa à temperatura de $65^{\circ} \mathrm{C}$ até massa constante.

Em ambos os cultivos, não foi necessário fazer nenhum controle fitossanitário. Nos períodos de baixa precipitação pluvial, durante os cultivos, foi feita a irrigação com água deionizada. A água foi adicionada vagarosamente até o início do escorrimento pelo orifício de drenagem, localizado na parte inferior do vaso.

A matéria seca da parte aérea de ambos os cultivos foi pesada e moída. A determinação dos teores dos nutrientes, na parte aérea das plantas de milho e soja, respectivamente, foi feita no extrato nítricoperclórico, conforme o método descrito por Tedesco et al. (1995).

Os teores de $\mathrm{Cu}$ e de $\mathrm{Zn}$ disponíveis no solo foram extraídos pelas soluções de $\mathrm{HCl} \mathrm{0,1} \mathrm{mol} \mathrm{L}^{-1}$ (Tedesco et al., 1995), de Mehlich-1 (Tedesco et al., 1995) e de Mehlich-3 (Mehlich, 1984). A determinação foi feita por espectrofotometria de absorção atômica.

Foram estabelecidas regressões lineares entre os teores extraídos pelos métodos em estudo e a quantidade absorvida pelas culturas do milho (primeiro cultivo) e da soja (segundo cultivo). A capacidade preditiva para avaliação dos nutrientes disponíveis pelos diferentes métodos foi determinada, principalmente, pela análise dos coeficientes de determinação. A comparação da capacidade extrativa de $\mathrm{Cu}$ e de $\mathrm{Zn}$ do solo foi feita por correlação, para verificar o grau de associação entre as variáveis.

\section{RESULTADOS E DISCUSSÃO}

\section{Cobre}

\section{Comparação entre métodos de extração}

$\mathrm{O}$ teor de $\mathrm{Cu}$ do solo extraído pela solução de $\mathrm{M}_{1}$ não diferiu do extraído pela solução de $\mathrm{HCl}$ (Quadro 3). Em parte, isso se deve ao mesmo princípio químico de extração dos dois procedimentos. No caso da solução de $\mathrm{HCl}$, a mistura solo-solução é agitada por $30 \mathrm{~min}$, em agitador horizontal, e, para o método $\mathrm{M}_{1}$, o tempo de agitação é de apenas 5 min (Lindsay \& Cox, 1985). Mesmo com tempo diferente de agitação entre os dois procedimentos, não houve diferença entre os teores extraídos pelo $\mathrm{M}_{1}$ e $\mathrm{HCl}$. Outro fator que pode alterar o teor extraído é a concentração de $\mathrm{H}^{+}$da solução extratora, que é maior na solução de $\mathrm{HCl}$ do que na de $\mathrm{M}_{1}$ (Lindsay \& Cox, 1985), porém não sendo diferentes quanto aos teorees extraídos.

Os coeficientes de correlação entre os teores extraídos de $\mathrm{Cu}$ do solo com a solução de $\mathrm{HCl}$ e a solução de $M_{1}$ foram de $0,92^{* *}$ e $0,63^{* *}$, respectivamente, para o primeiro e segundo cultivos (Figura 1a,b). A dispersão dos pontos pode ser atribuída à alta variabilidade nas características físicas, químicas e mineralógicas dos solos em estudo. Os coeficientes de correlação obtidos foram estatisticamente significativos. No entanto, a observação detalhada da distribuição dos pontos indica que para alguns solos as soluções agem de forma distinta. Assim, nos pontos em destaque nas figura $1 \mathrm{a}, \mathrm{b}$, a solução de $\mathrm{HCl}$ teve capacidade de extrair, aproximadamente, três vezes o teor extraído pela solução de $\mathrm{M}_{1}$. Isso indica que uma das soluções para esses solos terá uma baixa capacidade preditiva, quando comparada ao extrator "planta". 
Quadro 3. Teor de cobre extraído do solo por diferentes métodos antes do primeiro (milho) e segundo (soja) cultivos, teor e conteúdo de cobre na parte aérea e produção de matéria seca do milho e da soja

\begin{tabular}{|c|c|c|c|c|c|c|}
\hline \multirow{2}{*}{$\begin{array}{c}\text { Unidade de } \\
\text { Mapeamento }\end{array}$} & \multicolumn{3}{|c|}{ Cobre no solo } & \multicolumn{2}{|c|}{ Cobre na planta } & \multirow{2}{*}{ Matéria seca } \\
\hline & $\mathrm{HCl}$ & Mehlich-1 & Mehlich-3 & Teor & Conteúdo & \\
\hline & 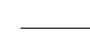 & $-\mathrm{mg} \mathrm{dm}^{-3}$ & - & $\mathrm{g} \mathrm{kg}^{-1}$ & $\mathrm{mg} /$ vaso & g/vaso \\
\hline & \multicolumn{6}{|c|}{ Cultura do milho } \\
\hline Cruz Alta & 2,3 & 5,0 & 3,6 & 3,3 & 0,52 & 15,52 \\
\hline Vacaria & 10,7 & 13,4 & 11,8 & 6,3 & 1,22 & 19,11 \\
\hline Uruguaiana & 5,1 & 9,5 & 7,9 & 3,3 & 0,42 & 12,55 \\
\hline Bom Jesus & 0,2 & 0,4 & 2,6 & 8,5 & 0,57 & 6,68 \\
\hline Bagé & 2,1 & 3,4 & 1,5 & 3,0 & 0,57 & 19,40 \\
\hline Aceguá & 2,4 & 2,3 & 4,0 & 3,0 & 0,48 & 16,15 \\
\hline São Jerônimo & 3,1 & 6,5 & 3,6 & 4,8 & 0,76 & 15,75 \\
\hline Passo Fundo & 4,1 & 6,9 & 4,4 & 3,7 & 0,45 & 12,11 \\
\hline Ciríaco & 5,2 & 5,3 & 7,1 & 3,7 & 0,78 & 21,11 \\
\hline Júlio de Castilhos & 4 & 7,2 & 3,8 & 3,7 & 0,66 & 17,76 \\
\hline Erechim & 7 & 9,5 & 8,5 & 6,7 & 1,03 & 15,59 \\
\hline \multirow[t]{2}{*}{ Estação } & 5,2 & 8,0 & 6,0 & 3,7 & 0,53 & 14,27 \\
\hline & \multicolumn{6}{|c|}{ Cultura da soja } \\
\hline Cruz Alta & 2,0 & 1,8 & 2,7 & 5,2 & 0,46 & 10,44 \\
\hline Uruguaiana & 3,8 & 3,8 & 5,6 & 6,3 & 0,77 & 14,77 \\
\hline Bagé & 2,2 & 1,2 & 1,8 & 5,9 & 0,52 & 9,56 \\
\hline São Jerônimo & 3,0 & 2,9 & 3,2 & 5,6 & 0,54 & 11,12 \\
\hline Passo Fundo & 3,5 & 3,3 & 3,8 & 5,9 & 0,63 & 13,27 \\
\hline Ciríaco & 4,7 & 2,3 & 6,9 & 7,1 & 0,90 & 15,02 \\
\hline Júlio de Castilhos & 3,3 & 2,1 & 3,5 & 4,8 & 0,57 & 16,91 \\
\hline Erechim & 5,5 & 4,1 & 7,9 & 7,8 & 1,00 & 13,17 \\
\hline Estação & 5,8 & 2,9 & 5,7 & 5,9 & 0,69 & 14,36 \\
\hline
\end{tabular}

Esses resultados corroboram os obtidos por Lantmann \& Meurer (1982) e Bataglia \& Raij (1989), principalmente quanto aos teores extraídos. Pereira et al. (2001), comparando essas duas soluções em 103 amostras da camada superficial de solos do Estado do Rio de Janeiro, obtiveram um coeficiente de correlação entre ambas de $0,75^{*}$, e os teores extraídos foram, aproximadamente, $25 \%$ menores com a solução de $\mathrm{M}_{1}$.

Os teores extraídos com a solução de $\mathrm{M}_{3}$ foram semelhantes àquelas com o $\mathrm{HCl}$, para os solos do primeiro e segundo cultivos (Figura 1c,d). A semelhança dos teores extraídos deve-se à composição química da solução de $\mathrm{M}_{3}$. Nessa, é incluído o reagente EDTA, que tem alta capacidade quelatizante (Abreu et al., 1996) em relação ao $\mathrm{Cu}$. Por esse processo, o $\mathrm{Cu}$ da solução é retirado e há formação de novo equilíbrio químico entre o $\mathrm{Cu}$ da solução e o retido na matéria orgânica do solo, principalmente; assim, mesmo com o tempo de agitação menor (5 min), extrai teor semelhante ao da solução de $\mathrm{HCl}$.
A relação entre os teorees extraídos de $\mathrm{Cu}$ pelas soluções de $\mathrm{HCl}$ e de $\mathrm{M}_{3}$ é mostrada na figura 1c,d. Os coeficientes de correlação foram elevados em ambos os cultivos $\left(0,94^{* *}\right.$ e $\left.0,89^{* *}\right)$. O alto grau de associação obtido deve-se à capacidade de extração semelhante das soluções em extraírem $\mathrm{Cu}$ de diferentes formas de $\mathrm{Cu}$ dos solos. A dispersão dos pontos na regressão foi menor que a observada na comparação do método $\mathrm{HCl}$ com o $\mathrm{M}_{1}$. A tendência de os coeficientes de correlação serem menores no segundo cultivo deve-se, em parte, ao menor número de solos utilizados para comparação.

\section{Teores extraídos pelas soluções e a absorção pelas plantas}

Os coeficientes de determinação entre o teor de $\mathrm{Cu}$ no solo, obtido com a solução de $\mathrm{HCl}$, e a quantidade de $\mathrm{Cu}$ absorvido pelas plantas foram de $0,56^{* *} \mathrm{e} 0,66^{* *}$, respectivamente, para o primeiro e o segundo cultivo (Figura 2a,b). Constatou-se baixo grau de semelhança para as duas culturas, mesmo havendo significância estatística para os coeficientes. Um coeficiente maior 

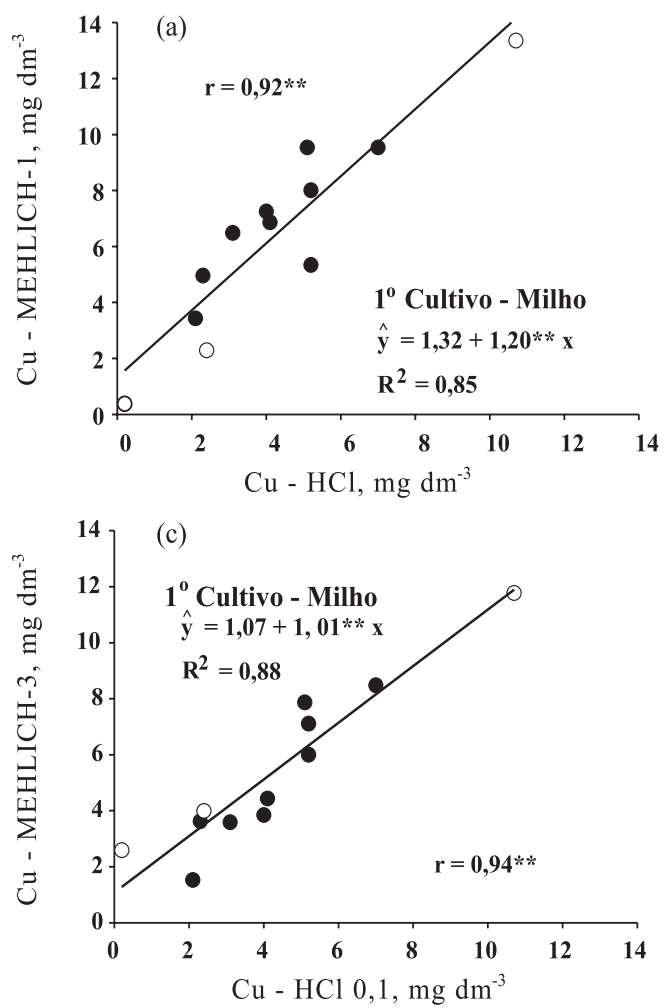
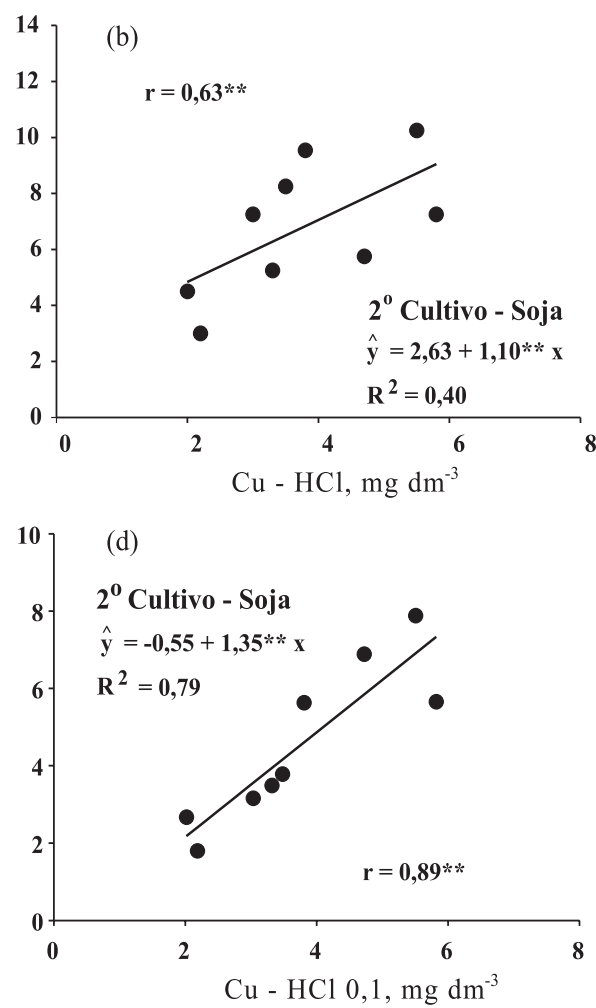

Figura 1. Relação entre os teores de cobre extraídos pelas soluções de HCl, Mehlich-1 e Mehlich-3, respectivamente, em amostras de solos coletadas antes do primeiro [(a) e (c)], com milho, e segundo cultivos [(b) e (d)], com soja.

obtido com a cultura da soja em relação ao obtido com o milho poderia indicar a influência da espécie utilizada como planta-teste. No entanto, essa afirmação não se sustenta, pois a mera inclusão ou retirada de um dos solos avaliados poderia alterar a grandeza dos coeficientes.

Em um estudo semelhante, com 26 solos do Estado de São Paulo (Bataglia \& Raij, 1989), utilizando as culturas de girassol e sorgo, os coeficientes de determinação obtidos entre os teores extraídos com a solução de $\mathrm{M}_{1}$ e a quantidade absorvida por essas culturas foram de 0,12 para o girassol e 0,17 para o sorgo, em amostras de solos não tratadas com calcário. Nas mesmas amostras de solos, porém tratadas com calcário, os coeficientes foram de 0,10 para o girassol e 0,15 para o sorgo. Os autores não informaram se isso se deveu a alguns poucos solos com características muito diferentes ou se a dispersão dos pontos foi normal, evidenciando a incapacidade da solução de $\mathrm{HCl}$ em estimar a disponibilidade $\mathrm{Cu}$ para as plantas.

A baixa relação obtida entre o $\mathrm{Cu}$ absorvido pelas plantas e os teores de $\mathrm{Cu}$ extraídos pela solução de $\mathrm{M}_{1}$ demonstra que o método não foi eficiente em avaliar a disponibilidade de $\mathrm{Cu}$ para o milho e para a soja em solos com amplas variações nas características químicas, físicas e mineralógicas (Figura 2c,d). A relação entre os teores extraídos de $\mathrm{Cu}$ do solo pela solução de $\mathrm{M}_{1}$ e as quantidades de $\mathrm{Cu}$ absorvido pelo milho é mostrada na figura $2 \mathrm{c}, \mathrm{d}$. Os coeficientes de determinação foram de $0,35^{*}$ e $0,43^{*}$, respectivamente, para o primeiro e segundo cultivos. Em um estudo de eficiência de extratores para micronutrientes em 26 solos do Estado de São Paulo, Bataglia \& Raij (1989) obtiveram coeficientes de determinação de 0,13 e 0,20 com as culturas de girassol e sorgo, respectivamente, para a mesma relação. Esses autores concluíram que a solução de $\mathrm{M}_{1}$ é eficiente na extração de $\mathrm{Cu}$ disponível para as plantas em solos do Estado de São Paulo. Não há informação de qual foi o critério para essa conclusão, pois os coeficientes pouco diferem dos obtidos com a solução de $\mathrm{HCl}$. Em outro estudo, realizado com oito solos do Estado do Rio Grande do Sul (Luchese, 1985), para avaliar a disponibilidade de $\mathrm{Cu}$ tendo como planta-teste aveia, foi obtido um coeficiente de determinação ainda menor $\left(R^{2}=0,01\right)$ do que os obtidos por Bataglia \& Raij (1989) e no presente trabalho. Há ainda o estudo de Abreu et al. (1996), no qual foram comparados vários extratores quanto à capacidade de avaliar a disponibilidade do $\mathrm{Cu}$ do solo tendo como planta-teste aveia e feijão, em amostras de 31 solos do Estado de São Paulo. O coeficiente de correlação obtido para a relação teor de $\mathrm{Cu}$ no solo, estimado pela solução de $\mathrm{M}_{1}$, e a quantidade de $\mathrm{Cu}$ acumulado pelas plantas foi de 0,45 , para ambas as culturas. Borkert et al. (2004) concluíram que a solução de $\mathrm{M}_{1}$ avalia adequadamente o $\mathrm{Cu}$ disponível às plantas. Isso, no entanto, pode ser devido à 

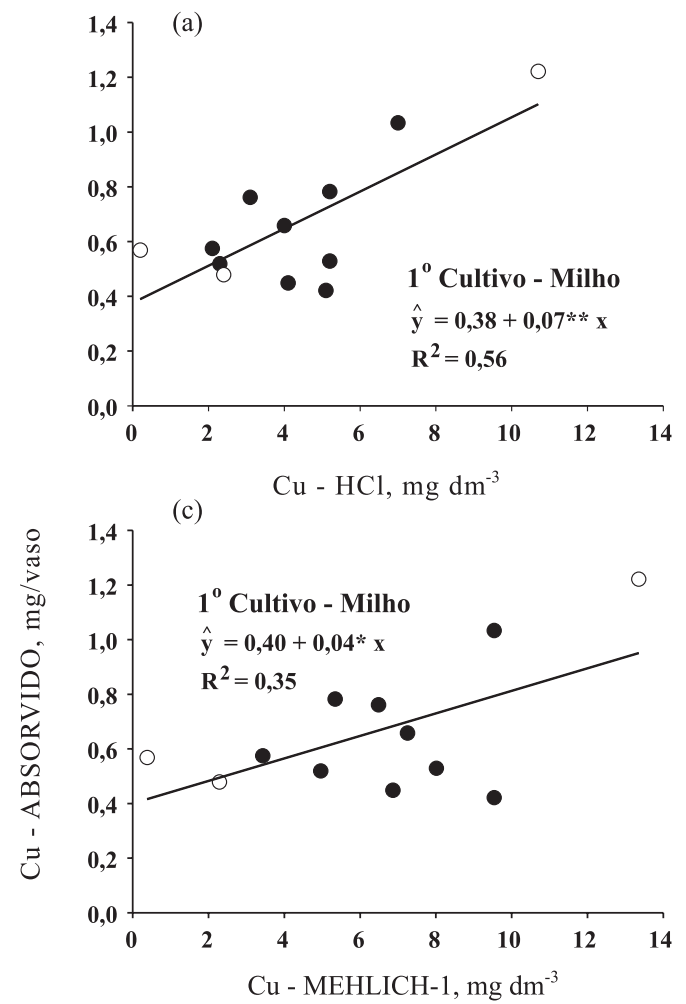

(e)

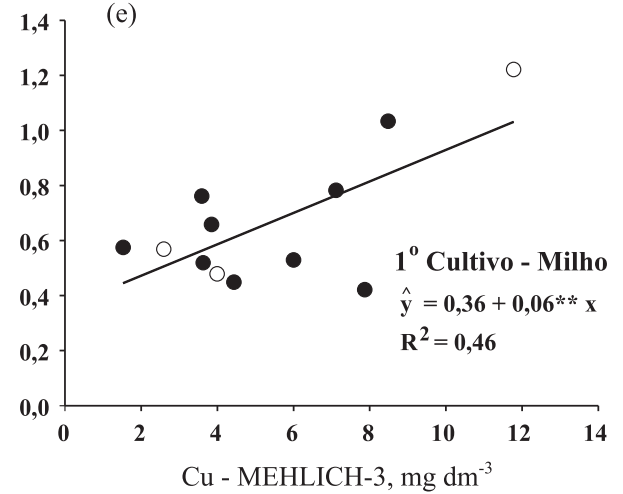

(b)

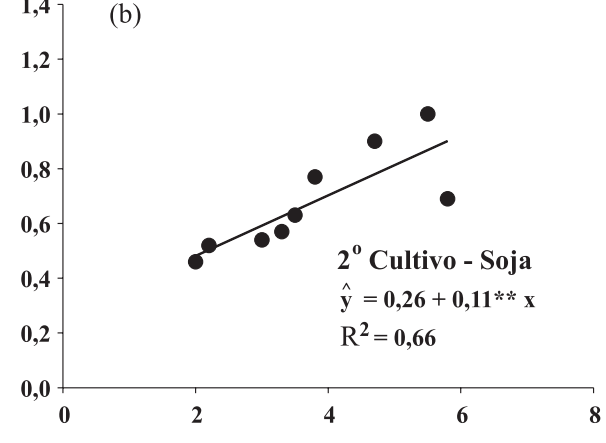

(d)

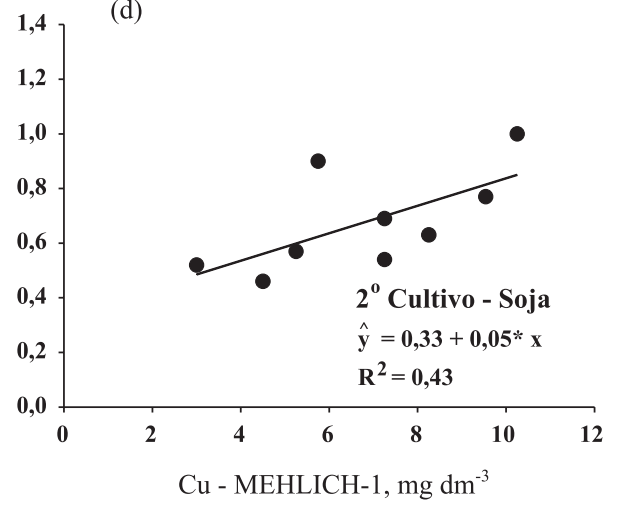

(f)

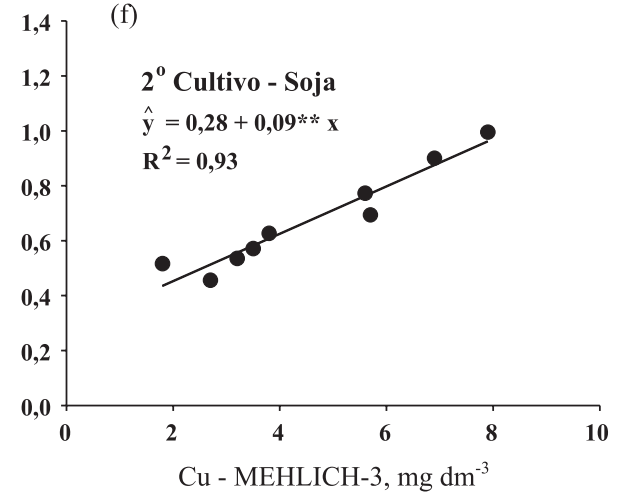

Figura 2. Relação entre os teores de cobre extraídos pelas soluções de HCl, Mehlich-1 e Mehlich-3, respectivamente, e a quantidade acumulada pelas plantas de milho [(a), (c) e (e)], no primeiro cultivo, e de soja [(b), (d) e (f)], no segundo cultivo.

utilização de doses crescentes de Cu em somente um tipo de solo, o que elimina a variabilidade existente entre solos diferentes.

A relação entre o teor de $\mathrm{Cu}$ no solo extraído pela solução de $\mathrm{M}_{3}$ e a quantidade de $\mathrm{Cu}$ absorvido é mostrada na figura $2 \mathrm{e}, \mathrm{f}$. Os coeficientes de determinação entre o teor de $\mathrm{Cu}$ no solo extraído pela solução de $\mathrm{M}_{3}$ e a quantidade de $\mathrm{Cu}$ acumulada pelas plantas foram de $0,46^{* *}$ e $0,93^{* *}$, respectivamente, para o primeiro e segundo cultivos (Figura 2e,f). As quantidades absorvidas e acumuladas pelas plantas foram semelhantes nos dois cultivos, demonstrando que a solução de $\mathrm{M}_{3}$ pode estimar com maior eficiência a disponibilidade de $\mathrm{Cu}$ para as plantas do que as soluções de $\mathrm{M}_{1}$ e de $\mathrm{HCl}$. Isso é verdadeiro, em parte, quando se utiliza somente o coeficiente de determinação para a comparação. No entanto, o maior coeficiente de determinação só foi verificado no segundo cultivo - situação na qual, no estudo de disponibilidade, foram excluídos três solos em relação ao primeiro estudo, conforme indicado anteriormente. É possível, portanto, que outros fatores, inclusive a cultura, interferiram nos resultados obtidos.

Silva et al. (2002) utilizaram a solução de $\mathrm{M}_{3}$ em um estudo de avaliação da disponibilidade de $\mathrm{Cu}$ em solos do Estado do Paraná e obtiveram um coeficiente de determinação de 0,37 entre os teores extraídos por essa solução e a quantidade de $\mathrm{Cu}$ absorvida por 
plantas de milho. Os solos utilizados eram arenosos, com acidez corrigida e com teores de matéria orgânica de 15 a $40 \mathrm{~g} \mathrm{dm}^{-3}$. Quando a regressão foi feita apenas com solos argilosos, com teores de matéria orgânica variando de 35 a $50 \mathrm{~g} \mathrm{dm}^{-3}$, esses autores obtiveram coeficiente de determinação de 0,72 . Eles constataram que o fator tipo de solo influenciou o desempenho do método. Borkert et al. (2004) também testaram essa solução e concluíram que o $\mathrm{M}_{3}$ é eficiente na avaliação da disponibilidade de $\mathrm{Cu}$ para as plantas. Como explicado anteriormente, esses autores utilizaram somente um solo com adição de doses crescentes de $\mathrm{Cu}$, o que dificulta a generalização dos resultados.

Em geral, pelos dados obtidos, a solução de $\mathrm{M}_{3}$ foi superior às outras soluções testadas. No primeiro cultivo há semelhança dos coeficientes de determinação das soluções de $\mathrm{HCl}$ e $\mathrm{M}_{3}$; no segundo, a capacidade preditiva foi superior com a solução de $\mathrm{M}_{3}$ do que com a de $\mathrm{HCl}$. Uma das explicações para a superioridade da solução de $\mathrm{M}_{3}$ em avaliar a disponibilidade de $\mathrm{Cu}$ do solo às plantas pode ser a presença de EDTA, por sua capacidade quelatizante. Abreu et al. (1996), em estudo para avaliar a eficiência de extratores multielementares em 31 solos do Estado de São Paulo, obtiveram um coeficiente de determinação de 0,80 para a solução de $\mathrm{M}_{3}$ e também concluíram que soluções com quelatizantes são mais eficientes na avaliação da disponibilidade, de $\mathrm{Cu}$ do solo para as plantas. Borkert et al. (2004) não obtiveram diferença entre as soluções de $\mathrm{M}_{3}\left(\mathrm{R}^{2}=0,90\right)$ e $\mathrm{M}_{1}\left(\mathrm{R}^{2}=0,90\right)$ na avaliação da disponibilidade de $\mathrm{Cu}$ para a soja, concluindo que ambas as soluções podem ser utilizadas. Por facilidade operacional no laboratório, a solução de $\mathrm{M}_{1}$ seria a mais indicada; nesta solução determinam-se também $\mathrm{P}$ e K.

\section{Zinco}

\section{Comparação entre métodos de extração}

Os teores de Zn extraídos pelos diferentes métodos são mostrados no quadro 4. A relação entre os teores de $\mathrm{Zn}$ extraídos pelas soluções de $\mathrm{HCl}$ e de $\mathrm{M}_{1}$ é mostrada na figura 3a,b. Os coeficientes de correlação obtidos foram de $0,93^{* *} 0,97^{* *}$ com solos do primeiro e segundo cultivos, respectivamente (Figura 3a,b). Constatou-se, pelos coeficientes angulares das retas de regressão, que os teores extraídos foram semelhantes. O coeficiente de correlação obtido foi

Quadro 4. Teores de zinco extraídos por diferentes métodos antes do primeiro (milho) e segundo (soja) cultivos, teor e conteúdo de cobre na parte aérea e produção de matéria seca do milho e da soja

\begin{tabular}{|c|c|c|c|c|c|c|}
\hline \multirow{2}{*}{$\begin{array}{l}\text { Unidade de } \\
\text { Mapeamento }\end{array}$} & \multicolumn{3}{|c|}{ Zinco no solo } & \multicolumn{2}{|c|}{ Zinco na planta } & \multirow{2}{*}{ Matéria seca } \\
\hline & $\mathrm{HCl}$ & Mehlich-1 & Mehlich-3 & Teor & Conteúdo & \\
\hline & $\longrightarrow$ & $-\mathrm{mg} \mathrm{dm}^{-3}$ & - & $\mathrm{g} \mathrm{kg}^{-1}$ & $\mathrm{mg} /$ vaso & $\mathrm{g} /$ vaso \\
\hline & \multicolumn{6}{|c|}{ Cultura do milho } \\
\hline Cruz Alta & 2,3 & 2,6 & 2,5 & 11,3 & 1,75 & 15,52 \\
\hline Vacaria & 6,7 & 8,2 & 4,5 & 31,7 & 6,00 & 19,11 \\
\hline Uruguaiana & 4,5 & 4,7 & 3,9 & 18,5 & 2,31 & 12,55 \\
\hline Bom Jesus & 0,7 & 0,1 & 2,0 & 14,4 & 0,96 & 6,68 \\
\hline Bagé & 2,6 & 3,6 & 3,8 & 23,5 & 4,62 & 19,40 \\
\hline Aceguá & 5,6 & 5,4 & 5,1 & 16,7 & 2,71 & 16,15 \\
\hline São Jerônimo & 3,6 & 4,1 & 3,2 & 24,5 & 3,85 & 15,75 \\
\hline Passo Fundo & 4,1 & 3,8 & 3,5 & 12,7 & 1,54 & 12,11 \\
\hline Ciríaco & 7,8 & 6,3 & 4,6 & 18,3 & 3,86 & 21,11 \\
\hline Júlio de Castilhos & 6,0 & 5,9 & 4,7 & 16,8 & 3,01 & 17,76 \\
\hline Erechim & 6,0 & 6,6 & 4,5 & 12,2 & 1,89 & 15,59 \\
\hline \multirow[t]{2}{*}{ Estação } & 6,0 & 6,8 & 6,0 & 16,9 & 2,41 & 14,27 \\
\hline & \multicolumn{6}{|c|}{ Cultura da soja } \\
\hline Cruz Alta & 1,4 & 0,7 & 2,2 & 41,1 & 4,29 & 10,44 \\
\hline Uruguaiana & 3,9 & 3,6 & 3,3 & 42,9 & 6,33 & 14,77 \\
\hline Bagé & 4,1 & 4,9 & 3,8 & 54,3 & 5,19 & 9,56 \\
\hline São Jerônimo & 2,8 & 3,1 & 3,1 & 63,4 & 7,04 & 11,12 \\
\hline Passo Fundo & 3,1 & 2,9 & 3,4 & 44,1 & 5,85 & 13,27 \\
\hline Ciríaco & 6,1 & 5,8 & 4,3 & 50,8 & 7,62 & 15,02 \\
\hline Júlio de Castilhos & 3,9 & 3,7 & 3,4 & 47,2 & 7,99 & 16,91 \\
\hline Erechim & 5,5 & 5,1 & 3,7 & 46,9 & 6,16 & 13,17 \\
\hline Estação & 7,5 & 6,9 & 5,7 & 52,6 & 7,54 & 14,36 \\
\hline
\end{tabular}


semelhante ao encontrado por Bataglia \& Raij (1989) em 26 solos paulistas $(r=0,90)$, sem a adição de $\mathrm{Zn}$ aos solos. Em outro estudo, Bataglia \& Raij (1994), utilizando os mesmos solos que Bataglia \& Raij (1989) na avaliação da disponibilidade de Zn, obtiveram alto grau de associação entre os teores de Zn extraídos pelas soluções de $\mathrm{HCl}$ e $\mathrm{M}_{1}(\mathrm{r}=0,99)$. O maior coeficiente de correlação obtido por esses autores, em relação ao obtido neste trabalho, deve-se, em parte, ao tempo de agitação das amostras. Bataglia \& Raij (1994) utilizaram o tempo de 30 min de agitação para ambas as soluções, diferente dos 5 min utilizados para a solução de $\mathrm{M}_{1}$ neste trabalho. Em outra comparação das mesmas soluções, Buzetti (1992), utilizando nove solos do Estado de São Paulo, obteve o coeficiente de correlação de 0,98 .

Os resultados obtidos neste trabalho contrastam com os obtidos por Lantmann \& Meurer (1982). Esses autores, para a mesma relação, obtiveram o coeficiente de correlação de 0,73 num estudo com 10 solos do RS. Aos solos não foram adicionados calcário ou doses de $\mathrm{Zn}$. Além do coeficiente menor, os teores extraídos pela solução de $\mathrm{M}_{1}$ foram, aproximadamente, $50 \%$ menores que as obtidas com a solução de HCl. Após a aplicação do calcário (para pH 6,0), as soluções extraíram menores teores de $\mathrm{Zn}$ dos solos; possivelmente, isso esteja associado à maior adsorção de Zn pelos solos (Roesch, 1979), às reações das soluções extratoras com carbonatos e, ou, bicarbonatos livres (Nelson et al., 1959) e ao tempo de equilíbrio utilizado na extração, que pode ter sido insuficiente. Também contrastam com os de Pereira et al. (2001) em Estudo com 103 amostras de solo do estado do Rio de Janeiro. Esses autores obtiveram um coeficiente de correlação de 0,87 entre os teores extraídos com as mesmas soluções e informaram que a solução com maior capacidade extrativa foi o $\mathrm{HCl}$. Contudo, eles não indicaram o percentual em relação à solução de $\mathrm{M}_{1}$, o que dificulta o estabelecimento de uma comparação mais detalhada com os dados obtidos. Oliveira et al. (1999), em 12 amostras de solos sem adição de Zn, obtiveram coeficientes de correlação de 0,98 e 0,88 sem e com a adição de $\mathrm{Zn}$, respectivamente. Possivelmente, a forma de $\mathrm{Zn}$ poderia influir no desempenho de uma das soluções. Os autores não comentaram a respeito nem informaram se os teores extraídos foram semelhantes para ambas as soluções. Isso seria mais um fator a considerar na escolha de um método de avaliação da disponibilidade de $\mathrm{Zn}$ às plantas.

A relação entre os teores extraídos pelas soluções de $\mathrm{HCl}$ e $\mathrm{M}_{3}$ é mostrada na figura 3c,d. Os coeficientes de correlação entre os teores dos solos amostrados antes do primeiro e do segundo cultivo foram de $0,84^{* *} \mathrm{e}$ $0,94^{* *}$, respectivamente (Figura $3 \mathrm{c}, \mathrm{d}$ ). Os teores de Zn extraídos pela solução de $\mathrm{M}_{3}$ corresponderam, em média, a $89 \%$ dos extraídos com a solução de $\mathrm{HCl}$. No entanto, por esses dados, a substituição de uma solução por outra com ajuste pela equação não é adequada. Como a reta de ajuste linear não passa pela origem, ao se converter um valor obtido pela (a)

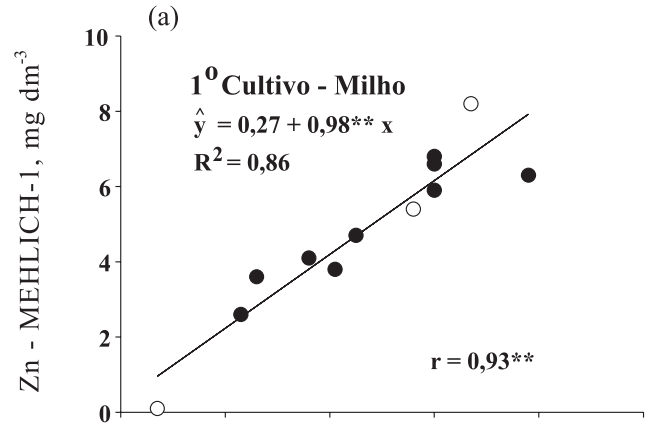

(c)

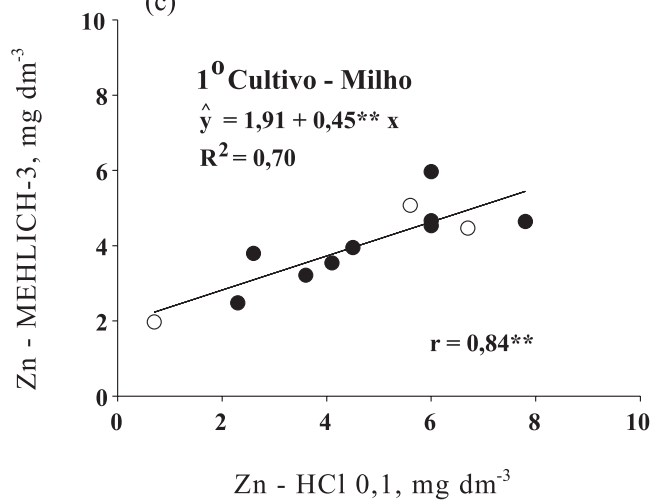

(b)

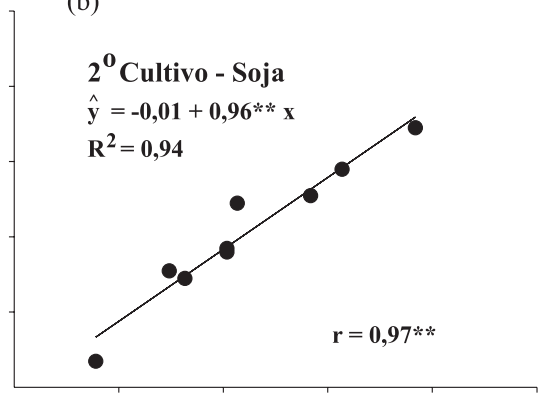

(d)

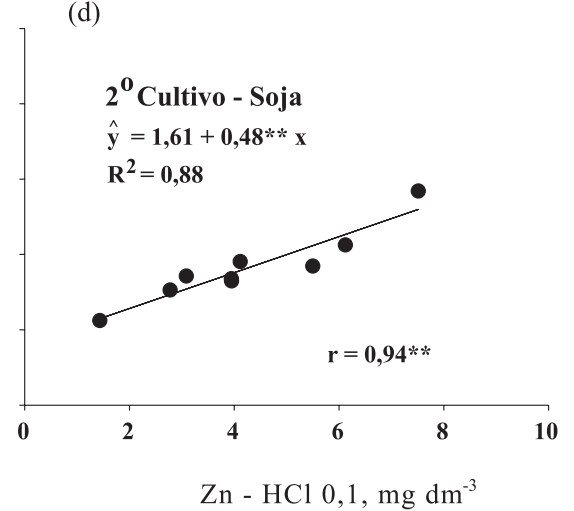

Figura 3. Relação entre os teores de zinco extraídos pelas soluções de HCl, Mehlich-1 e Mehlich-3, respectivamente, em amostras de solos coletadas antes do primeiro [(a) e (c)], com milho, e segundo cultivos [(b) e (d)], com soja. 
solução $\mathrm{M}_{3}$ para a solução de $\mathrm{HCl}$, ele será subestimado para valores baixos e superestimado para valores altos. Os coeficientes mais altos obtidos com solos do segundo cultivo, possivelmente, estejam associados ao menor número de solos utilizados, já que alguns destes foram excluídos das relações por apresentarem alguma(s) característica(s) que poderia(m) ser limitante(s) ao crescimento e desenvolvimento da soja. Em geral, os resultados obtidos na comparação da capacidade extrativa das soluções de $\mathrm{HCl}$ e de $\mathrm{M}_{3}$ concordam com aqueles de muitos trabalhos publicados sobre o mesmo assunto (Oliveira et al.,1999; Borkert et al., 2004).

Relação entre as quantidades extraídas pelas soluções e a absorção pelas plantas

Os coeficientes de determinação obtidos entre os teores de Zn extraídos do solo com a solução de $\mathrm{HCl}$ e a quantidade absorvida pelas plantas de milho e de soja foram de $0,18^{*}$ e $0,37^{*}$ para o primeiro e segundo cultivos, respectivamente (Figura 4a,b). Esses valores dos coeficientes destoam dos apresentados na literatura. Bataglia \& Raij (1989), utilizando 26 solos do Estado de São Paulo, obtiveram um coeficiente de determinação de 0,55 para a mesma relação com a cultura de girassol. No entanto, quando cultivaram sorgo na sequência, o coeficiente obtido foi de 0,27 . Isso poderia, em parte, indicar a influência do tipo de planta utilizada, mas também pode ser devido a outros fatores que se alteram no tempo, ou seja, entre um e outro cultivo. Em geral, na maioria dos trabalhos encontrados na literatura (Lantmann \& Meurer, 1982; Buzzeti, 1992; Bataglia \& Raij, 1994), os coeficientes de determinação são maiores do que os obtidos neste estudo.
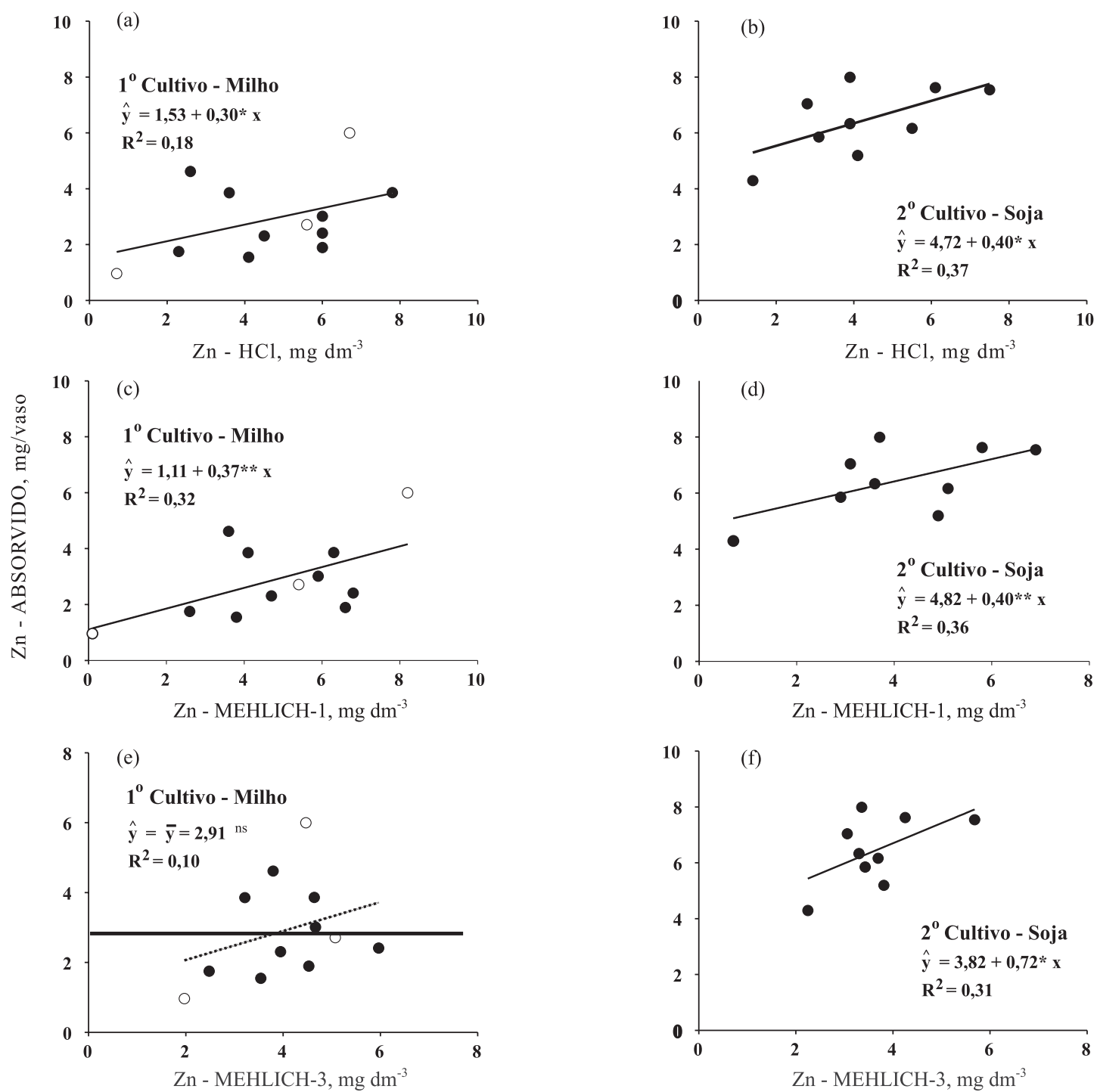

Figura 4. Relação entre os teores de zinco extraídos pelas soluções de HCl, Mehlich-1 e Mehlich-3, respectivamente, e a quantidade acumulada pelas plantas de milho [(a), (c) e (e)], no primeiro cultivo, e de soja [(b), (d) e (f)], no segundo cultivo. 
Os coeficientes de determinação obtidos entre as quantidades de $\mathrm{Zn}$ absorvidas pelo milho e soja e os teores extraídos pela solução de $\mathrm{M}_{1}$ foram de $0,32^{*} \mathrm{e}$ $0,36^{*}$ para o primeiro e segundo cultivos, respectivamente (Figura 4c,d). Em vários outros estudos de comparação de métodos, a solução de $\mathrm{M}_{1}$ não têm sido satisfatória para avaliar a disponibilidade de $\mathrm{Zn}$ do solo para as plantas. Nos trabalhos de Bataglia \& Raij (1989) e Bataglia \& Raij (1994), os coeficientes de determinação variaram de 0,22 a 0,28 em cultura como sorgo e milho. Em outros estudos (Lantmann \& Meurer, 1982; Bataglia \& Raij, 1989; Buzetti, 1992), foram obtidos coeficientes de determinação de 0,58 a 0,73. O maior coeficiente foi obtido por Buzetti (1992) que utilizou um pequeno número de solos e adicionou doses crescentes de $\mathrm{Zn}$, diminuindo assim a variabilidade nas características físicas, químicas e mineralógicas.

A relação linear entre os teores de Zn extraídos pela solução de $\mathrm{M}_{3}$ e as quantidades de $\mathrm{Zn}$ absorvidas apresentaram coeficientes de determinação de $0,10^{\text {ns }}$ e $0,31^{*}$, respectivamente, para os cultivos de milho e soja (Figura 4e,f). Pode-se inferir que grande parte do Zn não está contida na matéria orgânica e sim na fase mineral. Como todas as soluções são ácidas e têm princípios semelhantes de ação, a presença do EDTA na solução de $\mathrm{M}_{3}$ não foi vantajosa na melhoria da estimativa e da disponibilidade desse nutriente. Esses resultados concordam com os obtidos por Davis et al. (1995) em estudo com solos da Geórgia, utilizando o amendoim como planta-teste e somente um solo para o estudo. O coeficiente obtido foi de 0,14 . Um coeficiente maior para essa mesma relação foi obtido por Rodrigues et al. (2002), estudando métodos de avaliação da disponibilidade de Zn para 10 solos paranaenses.

\section{CONCLUSÃO}

1. As soluções de $\mathrm{HCl} 0,1 \mathrm{~mol} \mathrm{~L}^{-1}$, de Mehlich-1 e de Mehlich-3 não avaliam adequadamente a disponibilidade de Cu e Zn para as plantas em solos do sul do Brasil. Entretanto, para avaliação da disponibilidade de $\mathrm{Cu}$ às plantas, a solução de Mehlich3 é a melhor alternativa.

2. As soluções testadas têm baixa eficiência na avaliação da disponibilidade de Zn para as plantas, porém a solução de Mehlich-1 tem vantagem pela facilidade de adaptação no laboratório de análise de solo, pois é a mesma solução utilizada para análise de $\mathrm{Pe}$ $\mathrm{K}$, não necessitando de adequação física ao laboratório.

\section{LITERATURA CITADA}

ABREU, C.A. \& RAIJ, B.van. Efeito da reação do solo no zinco extraído pelas soluções de DTPA e Mehlich - 1. Bragantia, 55:357-363, 1996.
ABREU, C.A.; RAIJ, B.van; ABREU, M.F.; SANTOS, W.R. \& ANDRADE, J.C. Efficiency of multinutrient extractants for the determination of available copper in soils. Comm. Soil Sci. Plant Anal., 27:763-771, 1996.

BARBOSA FILHO, M.P.; DYNIA, J.F. \& ZIMMERMANN, F.J.P. Resposta do arroz de sequeiro ao zinco e ao cobre com efeito residual para o milho. R. Bras. Ci. Solo, 14:333-338. 1990.

BATAGLIA, O. \& RAIJ, B.van. Eficiência de extratores de micronutrientes na análise de solo. R. Bras. Ci. Solo,13:205-212, 1989.

BATAGLIA, O. \& RAIJ, B.van. Soluções extratoras na avaliação da fitodisponibilidade de zinco em solos. R. Bras. Ci. Solo, 18:454-461, 1994.

BORKERT. C.M.; SFREDO, G.J.; ORTIZ, F.R. \& BRITO, O.R. Extratores para zinco, cobre e manganês em Latossolo Vermelho distrófico. In: REUNIÃO DA PESQUISA DE SOJA DA REGIÃO SUL, 32., Passo Fundo, 2004. Atas e Resumos. Passo Fundo, Embrapa Trigo, 2004. p.70-71.

BUZZETI, S. Estudo da eficiência de extratores químicos de zinco, no solo, para o milho. R. Bras. Ci. Solo, 16:367-372, 1992.

CANCELA, R.C.; FREIRE, A.R.; ABREU, C.A. \& GONZÁLEZ, A.P. Eficacia de cuatro extractantes em la evaluación de la disponibilidad de cobre para maíz y soja. Bragantia, 60:205-212, 2001.

COMISSÃO DE FERTILIDADE DO SOLO - CFSRS/SC. Manual de adubação e calagem para os Estados do Rio Grande do Sul e Santa Catarina. 10.ed. Porto Alegre, SBCS - NRS/UFRGS, 2004. 400p.

DAVIS, J.G.; GAINES, T.P. \& PARKER, M.B. Comparison of soil zinc extractants for detection of applied zinc and prediction of leaf concentration. Comm. Soil Sci. Plant Anal., 26:2969-2981, 1995.

EMPRESA BRASILEIRA DE PESQUISA AGROPECUÁRIA EMBRAPA. Centro Nacional de Pesquisa de Solos. Sistema brasileiro de classificação de solos. Rio de Janeiro, 1999.338p.

KROTH, P.L. Disponibilidade de fósforo no solo para as plantas e fatores que afetam a extração por resina de troca em membranas. Porto Alegre, Universidade Federal do Rio Grande do Sul, 1998. 167p. (Tese de Mestrado)

LANTMANN, A.F. \& MEURER, E.J. Estudo da eficiência de extratores para avaliação da disponibilidade do zinco disponível do solo para o milho. R. Bras. Ci. Solo, 6:131135,1982

LINDSAY, W.L. \& COX, F.R. Micronutrients soil testing for the tropics. In: VLEK, D.L.G., ed. Micronutrients in tropical food crop production: Developments in plant and soil sciences. Dordrecht, M. Vighoff, 1985. v.14. p.169200 .

LUCHESE, E.B. Disponibilidade de cobre e zinco para as plantas nos solos do Rio Grande do Sul. Porto Alegre, Universidade Federal do Rio Grande do Sul, 1985. 106p. (Tese de Mestrado) 
MEHLICH, A. Determination of $\mathrm{P}, \mathrm{Ca}, \mathrm{Mg}, \mathrm{K}, \mathrm{Na}$ and $\mathrm{NH} 4$ by North Carolina soil testing laboratories. Raleigh, University of North Carolina, 1953. 8p.

MEHLICH, A. Mehlich-3 soil test extractant: A modification of Mehlich 2 extractant. Comm. Soil Sci. Plant Anal., 15:14091416,1984

NELSON, W.L.; BROWN, F.C. \& VIETS, L.C. A method for assessing zinc status of soils using acid- extractable zinc and titratable alkalinity values. Soil Sci., 88:275$283,1959$.

OLIVEIRA, M.F.G.; NOVAIS, R.F.; NEVES, J.C.L.; VASCONCELLOS, C.A. \& ALVES, V.M.C. Relação entre o zinco "disponível" por diferentes extratores, e as frações de zinco em amostras de solos. R. Bras. Ci. Solo, 23:827836,1999 .

PEREIRA, M.G.; PÉREZ, D.V.; VALLADARES, G.S.; SOUZA, J.M.P.F. \& ANJOS, L.H.C. Comparação de métodos de extração de cobre, zinco, ferro e manganês em solos do Rio de Janeiro. R. Bras. Ci. Solo, 25:655-660, 2001.
RODRIGUES, M.R.L.; MALAVOLTA, E. \& MOREIRA, A. Avaliação de extratores de zinco em solos da Amazônia. In: FERTBIO 2002, Rio de Janeiro. Resumos. Rio de Janeiro, 2002. 4p. CD-ROM.

ROESCH, V. Fatores que afetam a adsorção de zinco em solos. Porto Alegre, Universidade Federal do Rio Grande do Sul,1979. 89p. (Tese de Mestrado)

SILVA, M.A.G.; MUNIZ, A.S. \& SENGIK, E. Disponibilidade de micronutrientes em solos do Paraná: Zinco e cobre. In: FERTBIO, 2002, Rio de Janeiro. Resumos. Rio de Janeiro, 2002. 4p. CD-ROM.

TEDESCO, M.J.; GIANELLO, C.; BISSANI, C.A.; BOHNEN, H. \& VOLKWEISS, S.J. Análise de solo, plantas e outros materiais. 2.ed. Porto Alegre, Universidade Federal do Rio Grande do Sul,, 1995. 147p. (Boletim Técnico, 5)

WEAR, J.I. \& SOMMER, A.L. Acid extractable zinc of soils in relation to the occurrence of zinc deficiency symptoms of corn: A method of analysis. Soil Sci. Soc. Am. Proc., 12:143144, 1948. 